\title{
ALMA observations of anisotropic dust mass loss in the inner circumstellar environment of the red supergiant VY Canis Majoris ${ }^{\star}$
}

\author{
E. O'Gorman ${ }^{1}$, W. Vlemmings ${ }^{1}$, A. M. S. Richards ${ }^{2}$, A. Baudry ${ }^{3,4}$, E. De Beck ${ }^{1}$, L. Decin ${ }^{5}$, G. M. Harper ${ }^{6}$, \\ E. M. Humphreys ${ }^{7}$, P. Kervella ${ }^{8,9,10}$, T. Khouri ${ }^{11}$, and S. Muller ${ }^{1}$ \\ ${ }^{1}$ Department of Earth and Space Sciences, Chalmers University of Technology, Onsala Space Observatory, 43992 Onsala, Sweden \\ e-mail: eamon.ogorman@chalmers.se \\ 2 Jodrell Bank Centre for Astrophysics, School of Physics and Astronomy, University of Manchester, Manchester M13 9PL, UK \\ 3 Univ. Bordeaux, LAB, UMR 5804, 33270 Floirac, France \\ ${ }^{4}$ CNRS, LAB, UMR 5804, 33270 Floirac, France \\ 5 Instituut voor Sterrenkunde, Katholieke Universiteit Leuven, Celestijnenlaan 200D, 3001 Leuven, Belgium \\ ${ }^{6}$ School of Physics, Trinity College Dublin, 2 Dublin, Ireland \\ 7 ESO, Karl-Schwarzschild-Str. 2, 85748 Garching, Germany \\ 8 LESIA, Observatoire de Paris, CNRS, UPMC, Université Paris-Diderot, PSL, 5 place Jules Janssen, 92195 Meudon, France \\ 9 UMI-FCA, CNRS/INSU, France (UMI 3386) \\ 10 Dept. de Astronomía, Universidad de Chile, Casilla 26-D Santiago, Chile \\ 11 Astronomical Institute Anton Pannekoek, University of Amsterdam, PO Box 94249, 1090 GE Amsterdam, The Netherlands
}

Received 2 October 2014 / Accepted 14 November 2014

\begin{abstract}
The processes leading to dust formation and the subsequent role it plays in driving mass loss in cool evolved stars is an area of intense study. Here we present high resolution ALMA Science Verification data of the continuum emission around the highly evolved oxygen-rich red supergiant VY CMa. These data enable us to study the dust in its inner circumstellar environment at a spatial resolution of 129 mas at $321 \mathrm{GHz}$ and 59 mas at $658 \mathrm{GHz}$, thus allowing us to trace dust on spatial scales down to $11 R_{\star}$ (71 AU). Two prominent dust components are detected and resolved. The brightest dust component, $\mathrm{C}$, is located 334 mas $\left(61 R_{\star}\right)$ southeast of the star and has a dust mass of at least $2.5 \times 10^{-4} M_{\odot}$. It has a dust emissivity spectral index of $\beta=-0.1$ at its peak, implying that it is optically thick at these frequencies with a cool core of $T_{\mathrm{d}} \lessgtr 100 \mathrm{~K}$. Interestingly, not a single molecule in the ALMA data has emission close to the peak of this massive dust clump. The other main dust component, VY, is located at the position of the star and contains a total dust mass of $4.0 \times 10^{-5} M_{\odot}$. It also contains a weaker dust feature extending over $60 R_{\star}$ to the north with the total component having a typical dust emissivity spectral index of $\beta=0.7$. We find that at least $17 \%$ of the dust mass around VY CMa is located in clumps ejected within a more quiescent roughly spherical stellar wind, with a quiescent dust mass loss rate $\mathrm{of}^{5} \times 10^{-6} M_{\odot} \mathrm{yr}^{-1}$. The anisotropic morphology of the dust indicates a continuous, directed mass loss over a few decades, suggesting that this mass loss cannot be driven by large convection cells alone.
\end{abstract}

Key words. supergiants - stars: winds, outflows - circumstellar matter - stars: individual: VY CMa - stars: evolution - stars: late-type

\section{Introduction}

Cool evolved stars are the main producers of dust in galaxies and are important drivers of the chemical evolution of matter in the Universe. Despite the importance of dust in a broad range of astrophysical phenomena, the conditions that lead to its formation in the outflows of evolved stars and its subsequent role in driving mass loss remain largely unknown. Oxygen-rich red supergiants (RSGs) are sources of inorganic dust (silicate and alumina) that is formed and plays a role in launching mass loss in the inner circumstellar environment of these stars. However, the dust properties in this region such as density, composition, and morphology have remained largely unknown because the spatial resolution is not adequate.

A prime target for studying the properties of dust around evolved stars is the enigmatic oxygen-rich RSG VY Canis Majoris (VY CMa). VY CMa is a mid-M spectral type (M5e Ia; Humphreys 1974) RSG with an extremely high mass-loss rate ( $\dot{M} \sim 3 \times 10^{-4} M_{\odot} \mathrm{yr}^{-1}$; Danchi et al. 1994) and can undergo periods of even more intense mass loss (Humphreys et al. 2007). This mass loss is about two orders of magnitude greater than

* Appendices are available in electronic form at http://www . aanda.org the well known early-M spectral type RSGs, Betelgeuse and Antares. Consequently, VY CMa has a highly dense and dusty circumstellar envelope that obscures it and produces a reflection nebula at optical wavelengths (Humphreys et al. 2007). Thanks to its relative proximity ( $d=1.2_{-0.10}^{+0.13} \mathrm{kpc}$; Zhang et al. 2012) and high intrinsic luminosity $\left(L=3 \times 10^{5} L_{\odot}\right.$ using the photometry of Smith et al. 2001), the dust emission from the circumstellar envelope around VY CMa has been studied from optical to centimeter wavelengths (e.g., Lipscy et al. 2005; Muller et al. 2007). The dusty envelope consists of a diffuse and extended region with loops and arcs expanding over several arc seconds through a more uniform medium. At the sub-arc-second level, the envelope consists of a dense and dusty central core, but little is known about it. It is within this region that the dust condenses and radiation pressure on dust helps drive mass-loss. In this Letter we report the results of a sub-100 mas study of this region using ALMA Science Verification data.

\section{Observations and results}

VY CMa was observed at $321 \mathrm{GHz}, 325 \mathrm{GHz}$, and $658 \mathrm{GHz}$ as part of the ALMA Science Verification process on 2013 16-19 August, using 16 to 20 antennas of the main array with projected baselines ranging from $14 \mathrm{~m}$ to $2.7 \mathrm{~km}$. Details of the 
Table 1. ALMA continuum observations of VY CMa.

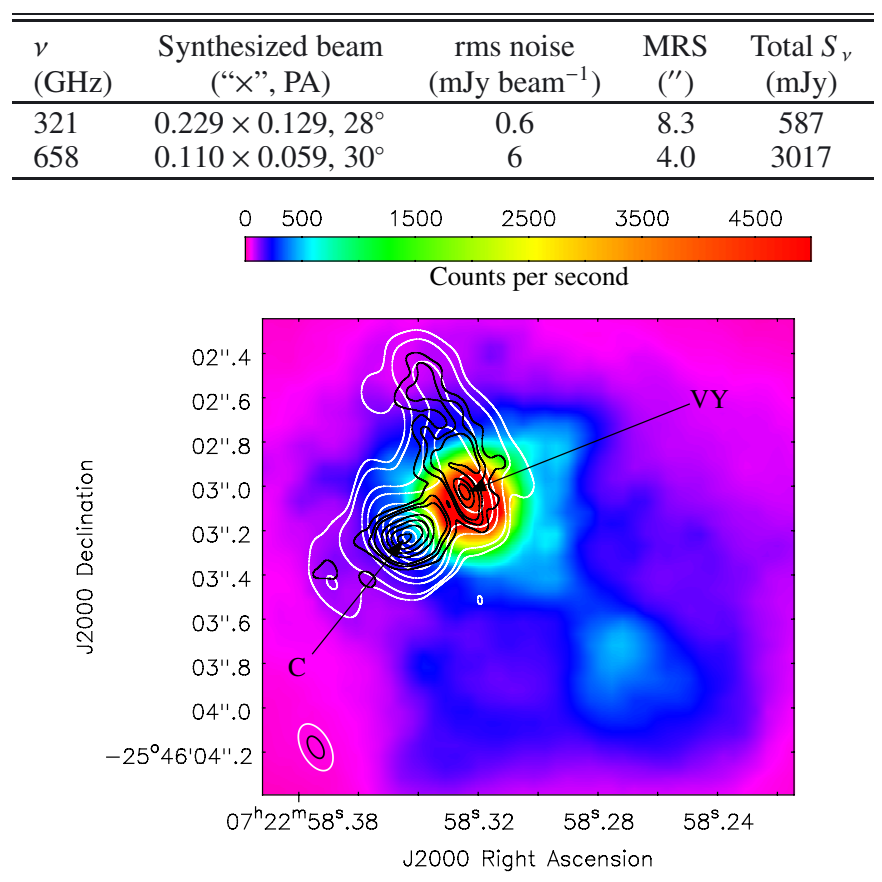

Fig. 1. HST/WFPC2 exposure of VY CMa using the F1042M filter (Smith et al. 2001) corrected for proper motion. The data has been truncated at 5000 counts per second and scaled with a power cycle of -1.5 to highlight the extended emission in the SW direction. The contours represent the ALMA data at $321 \mathrm{GHz}$ (white) and $658 \mathrm{GHz}$ (black) and the corresponding synthesized beams are located in the bottom left of the image. The $321 \mathrm{GHz}$ contour levels are set at $[5,10$, $30,50,100, \ldots ., 300] \times \sigma_{\text {rms }}$, while the $658 \mathrm{GHz}$ contour levels are set at $[3,6,9,20,30,40,50] \times \sigma_{\text {rms }}$.

observations and data processing are provided in Richards et al. (2014), hereafter $R+14$. For the purpose of the continuum analysis, we used a total of $1.74 \mathrm{GHz}$ line free continuum channels around $321 \mathrm{GHz}$ and $0.4 \mathrm{GHz}$ centered on $658 \mathrm{GHz}$. As the data around $325 \mathrm{GHz}$ provided similar results to the $321 \mathrm{GHz}$ data, but were affected by the $325.15 \mathrm{GHz}$ atmospheric water line resulting in increased noise, these data were not included in our subsequent analysis. The synthesized beam size, rms noise level, maximum recoverable scale (MRS), and total flux density of the two observing bands are given in Table 1 . The rms noise is not uniform across the field, most likely because of low surface brightness and resolved-out emission (see below). In Table 1 we report the rms in the region of maximum noise level, while in $\mathrm{R}+14$, the rms corresponds to the lower rms areas.

Uncertainties in the phase transfer of the data resulted in $\sim 110$ mas offsets between the main continuum peaks in the 321 and $658 \mathrm{GHz}$ images. To find the optimal (RA, Dec)-offset correction, we shifted the $658 \mathrm{GHz}$ image relative the $321 \mathrm{GHz}$ image and computed a 2D cross-correlation function for a range of shifts. The maximum correlation between the two images gave a ( -80 mas, -30 mas)-offset correction for the $658 \mathrm{GHz}$ image, similar to the correction found by $\mathrm{R}+14$ when aligning the peak position of the second brightest continuum component. The $321 \mathrm{GHz}$ image and the position corrected $658 \mathrm{GHz}$ image are plotted in white and black contours in Fig. 1, respectively. Continuous emission was detected above the $3 \sigma_{\text {rms }}$ level on scales as large as $1.4^{\prime \prime}$ at $321 \mathrm{GHz}$ and $0.8^{\prime \prime}$ at $658 \mathrm{GHz}$.

There are two prominent components and a weaker extended $\mathrm{N}-\mathrm{NE}$ feature that are reproduced at both frequencies, as seen in
Fig. 1. The position around the peak of the secondary component has recently been shown to coincide with the center of expansion of many maser emission lines and has been deduced to be the location of the star $(\mathrm{R}+14)$. This component (VY) connects to the weaker emission extending for $\sim 800$ mas at a position angle (PA, measured east of north) of $\sim 20^{\circ}$, which may contain some further substructure. Interestingly, the brightest component in both images $(\mathrm{C})$ is not at the location of the star itself but is 334 mas SE of the star in the plane of the sky. Using a distance of $1.2 \mathrm{kpc}$ to VY CMa, this angular distance corresponds to $400 \mathrm{AU}$, or $61 R_{\star}$ if a linear radius of $1420 R_{\odot}($ at $2 \mu \mathrm{m})$ is adopted (Wittkowski 2012). $\mathrm{R}+14$ have found that both blueand redshifted $325 \mathrm{GHz}$ and $658 \mathrm{GHz}$ masers, which are emitted out to $\sim 240 \mathrm{AU}$, straddle this main continuum component, so the projected distance between this component and the star is likely to be close to the actual distance.

We fit 2D elliptical Gaussian components to the two main continuum features and found that both the $\mathrm{C}$ and the VY components were resolved at both frequencies. The sizes and flux densities of these components were estimated from the deconvolved fits and are listed in Table A.1. The main C component is highly elongated at both frequencies in the SE direction, with a major-to-minor axis ratio, $\theta_{\text {maj }} / \theta_{\text {min }}=1.6 \pm 0.2$ at $321 \mathrm{GHz}$ and $\theta_{\text {maj }} / \theta_{\text {min }}=2.1 \pm 0.1$ at $658 \mathrm{GHz}$, with the axis of elongation pointing in the direction toward VY. We find that the size of component $\mathrm{C}$ is consistent at both frequencies within their errors. Comparing Tables 1 and A.1, we can see that $50 \%$ and $60 \%$ of the total continuum flux density emanates from the main $\mathrm{C}$ component at $321 \mathrm{GHz}$ and $658 \mathrm{GHz}$, respectively. The other main continuum component VY, is also resolved at both frequencies. It is elongated toward the North with $\theta_{\text {maj }} / \theta_{\text {min }}=1.5 \pm 0.4$ at $321 \mathrm{GHz}$ and $\theta_{\text {maj }} / \theta_{\text {min }}=2.1 \pm 0.5$ at $658 \mathrm{GHz}$, with emission extending to $27 R_{\star}$ (180 AU). It is centered on the location of the stellar photosphere, so it includes emission from the star itself at these sub-millimeter frequencies. The VY component contributes about $26 \%$ and $17 \%$ of the total ALMA continuum flux density at $321 \mathrm{GHz}$ and $658 \mathrm{GHz}$, respectively. The significant difference in size at the two frequencies cannot be attributed to the MRS on these small angular scales, but is probably due to a combination of different sensitivities to low surface brightness emission and the fact that the higher frequencies probe emission from hotter regions of VY.

A spectral index map of the continuum emission was created after convolving the $658 \mathrm{GHz}$ data with the synthesized beam from the $321 \mathrm{GHz}$ image, and regridding the convolved image to match the pixel scale of the $321 \mathrm{GHz}$ image. The resulting spectral index map is represented by the color image in Fig. 2, while the contour levels represent the convolved $658 \mathrm{GHz}$ image. All data below the $6 \sigma_{\text {rms }}$ noise level in the convolved $658 \mathrm{GHz}$ image have been omitted from Fig. 2. The middle region of the main continuum component $\mathrm{C}$ has a spectral index of $\alpha \sim 1.9$ (where $S_{v} \propto v^{\alpha}$ ). The spectral index value around the second continuum, or VY, and most of the extended emission in the $\mathrm{N}-\mathrm{NE}$ direction has a spectral index of $\alpha \sim 2.7$. The statistical error on the spectral index due to random noise errors is less than 0.2 in the $6 \sigma_{\mathrm{rms}}$ region and decreases to 0.05 in the peak region. The signal-to-noise ratio at $658 \mathrm{GHz}$ is less than that at $321 \mathrm{GHz}$, so this error is dominated by the $658 \mathrm{GHz}$ data. There is also a systematic absolute error of $\sim 0.22$ on the entire spectral index map based on the $\pm 15 \%$ uncertainty of the absolute flux calibration $(\mathrm{R}+14)$. We note that for VY, the difference in spectral index between the pixel flux densities in the map and what can be derived from the integrated flux densities is due to the smaller size of VY at $658 \mathrm{GHz}$. 


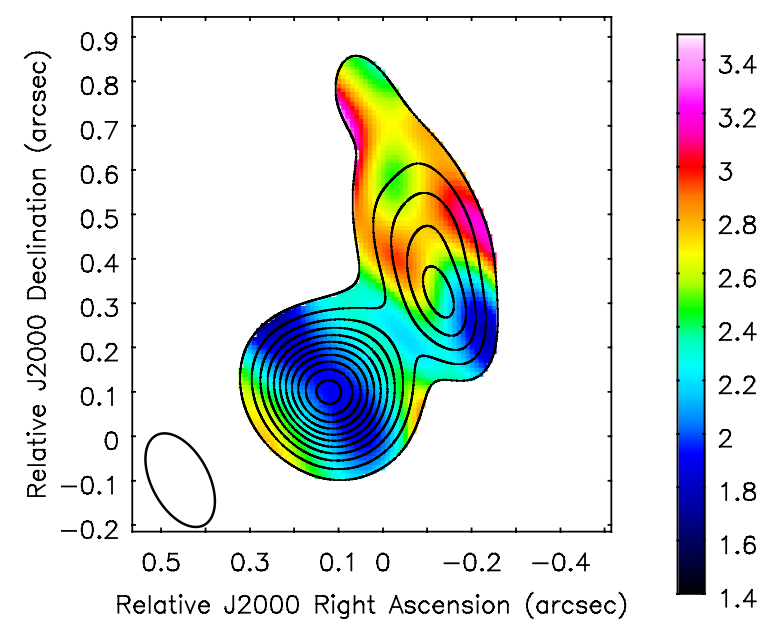

Fig. 2. Spectral index map derived from the $321 \mathrm{GHz}$ and $658 \mathrm{GHz}$ ALMA continuum maps. The strongest continuum component has a spectral index $\alpha=1.9 \pm 0.05$ at the peak, and the weaker elongated emission has a typical spectral index $\alpha=2.7 \pm 0.1$. The error values here do not include the systematic error based on the $\pm 15 \%$ uncertainty of the absolute flux calibration, which is in the same direction for both regions of the image. The contour map represents the $658 \mathrm{GHz}$ map, which was convolved with the $321 \mathrm{GHz}$ restoring beam to create the spectral index map. Contour levels are set at $[6,9, \ldots, 39] \times \sigma_{\text {rms. }}$.

\section{Discussion and conclusions}

\subsection{Comparison with HST}

The color image in Fig. 1 is an HST/WFPC2 exposure of VY CMa taken with the F1042M filter on 1999 March 22 (Smith et al. 2001). The data is adjusted for proper motion using the values of Zhang et al. (2012), and it can be seen that the peak emission fits the second main continuum component VY very well. The typical positional uncertainty rms in this HST image is 48 mas in RA and 146 mas in Dec, while the ALMA positional error is 35 mas, ruling out a correspondence between the C component in the ALMA images and the peak of the HST image. We can conclude that most of the escaping scattered light at optical wavelengths is emitted along the line of sight to the star itself. There is no evidence of the main C component in the HST image, probably because the HST emission does not trace dense dusty emission, but rather more tenuous regions in the circumstellar environment. Scattered light polarimetry with the HST does show a pronounced lower polarization toward the SE of the star (Jones et al. 2007), potentially indicating enhanced obscuration along the direction between the star and component $\mathrm{C}$.

The extended SW emission feature in the HST image, which may be a density cavity in the circumstellar environment formed by a previous episodic mass-loss event, has no detectable signature in our ALMA continuum images. This extended feature ends in what is named the SW clump in Smith et al. (2001) and Humphreys et al. (2007). This clump is thought to be related to an ejection event from the star and has been estimated to have a dust-mass lower limit of $5 \times 10^{-5} M_{\odot}$ and a temperature between $80 \mathrm{~K}$ and $210 \mathrm{~K}$ (Shenoy et al. 2013). However, we see no sign of this clump in the ALMA data. With our $3 \sigma_{\text {rms }}$ limits, we rule out a mass $>7 \times 10^{-6} M_{\odot}($ for $T=80 \mathrm{~K})$ or $>3 \times 10^{-6} M_{\odot}$ (for $T=210 \mathrm{~K}$ ). For the ALMA observations to be consistent with the lower limit estimates of Shenoy et al. (2013), the dust temperature would have to be $<20 \mathrm{~K}$. Alternatively, a different dust composition with different dust scattering and emissivity indices needs to be invoked. The exact nature of the SW clump is thus not clear. We also find no other compact submillimeter emission in the larger $\sim 8^{\prime \prime}$ region of dust seen with the HST.

\subsection{Properties of the dust}

Dust is the main source of the observed thermal submillimeter emission around VY CMa. If the dust is optically thin at these frequencies, and we assume the Rayleigh-Jeans approximation, then the observed flux density minus the stellar flux density contribution $S_{\star}$ is proportional to the mass of the emitting dust $M_{\mathrm{d}}$, such that

$S_{v}-S_{\star}=\frac{3 M_{\mathrm{d}} Q_{v} T_{\mathrm{d}} k v^{2}}{2 a_{\mathrm{g}} \rho_{\mathrm{g}} c^{2} d^{2}}$

where $Q_{v}$ is the grain emissivity, $a_{\mathrm{g}}$ and $\rho_{\mathrm{g}}$ are the radius and mass density of the dust grains, respectively, $d$ is the distance to the star, and $T_{\mathrm{d}}$ is the dust temperature (Knapp et al. 1993). Assuming that the grain emissivity has a power law dependence on frequency, $Q(v) \propto v^{\beta}$, then the flux density is $S_{v} \propto v^{\alpha}$, where $\alpha=2+\beta$. Here, $\beta$ is the dust emissivity spectral index and has typical values of $\beta=1.8 \pm 0.2$ for both diffuse and dense clouds in the interstellar medium (Draine 2006). For evolved stars, the dust emissivity index is generally lower (Knapp et al. 1993), and for VY CMa previous observations have found values between $\beta \sim 0-1.0$ (Shinnaga et al. 2004, Kamiński et al. 2013), while its circumstellar environment has been modeled using values of $\beta=0.9$ (Knapp et al. 1993).

Our spectral index map in Fig. 2 shows that the two spatially resolved continuum components in the inner circumstellar envelope have different spectral indices. The second brightest continuum component, VY, has a typical dust emissivity index of $\beta=0.7 \pm 0.1$ and is consistent with the previous singledish observations outlined in Appendix B. However, much of the main continuum component, $\mathrm{C}$, has a dust emissivity index of $\beta=-0.1$ around the location of the peak emission, meaning that the submillimeter emission from this component is, or is close to, becoming optically thick. Following Herman et al. (1986), optically thin dust at a radius $r_{\mathrm{d}}$ is heated by the incident stellar radiation field to a dust temperature

$T_{\mathrm{d}}=\left(\frac{L_{\star} T_{\star}^{\beta}}{16 \pi \sigma r_{\mathrm{d}}^{2}}\right)^{1 /(4+\beta)}$

where $L_{\star}$ and $T_{\star}$ are the stellar luminosity and temperature, respectively. Assuming $T_{\star}=3490 \mathrm{~K}$ (Wittkowski et al. 2012), the main continuum component, $\mathrm{C}$, would have an isothermal temperature of $450 \mathrm{~K}$ at $400 \mathrm{AU}$ from the star, while the VY component would have a value of $970 \mathrm{~K}$ at a mean dust radius of $67 \mathrm{AU}$. We stress that in deriving the temperature for the $\mathrm{C}$ component, we have assumed that the dust is optically thin even though the spectral index map indicates otherwise. We thus use $450 \mathrm{~K}$ as an upper limit to the temperature of $\mathrm{C}$. These rough estimates for the dust temperature allow us to empirically calculate the optical depth at both observing frequencies, the values of which are given in Table A.1. The VY component is optically thin at both frequencies with $\tau_{321-658 \mathrm{GHz}}=0.02-0.06$, while the main continuum component $\mathrm{C}$, has an optical depth of $\tau_{321-658 \mathrm{GHz}} \sim 0.2$, and would become optically thick at $110-120 \mathrm{~K}$ at both frequencies. Therefore, a cooler dust component in the center of $\mathrm{C}$ would explain the spectral index having a lower value than expected for optically thin dust. Alternatively, larger dust grains could also result in low $\beta$ values although our observing frequencies would then suggest that the dust grains would need to be much larger 
than $100 \mu \mathrm{m}$. Interestingly, we find that the thermal molecular emission lines from the ALMA data avoids the C component, as is consistent with the molecular emission offsets found in SMA observations (Muller et al. 2007; Kamiński et al. 2013). This could potentially support the low temperature and high optical depth suggestion because of low excitation and/or depletion onto dust grains.

Equation (1) can be re-arranged to find the total mass of dust in each of the two main continuum components. The main component $\mathrm{C}$ may not be fully optically thin at both frequencies and so its dust mass estimates will be lower limits. Following Knapp et al. (1993) we assume typical oxygen-rich star values for the radius and mass density of the dust grains, $a_{\mathrm{g}}=0.2 \mu \mathrm{m}$ and $\rho_{\mathrm{g}}=3.5 \mathrm{~g} \mathrm{~cm}^{-3}$. We slightly alter their grain emissivity function to include our derived dust emissivity spectral index so that $Q_{v}=5.65 \times 10^{-4}(v / 274.6 \mathrm{GHz})^{0.7}$. We also assume that the main $\mathrm{C}$ component has the same dust composition as VY, so the dust emissivity spectral index of $\mathrm{C}$ would also be $\beta=0.7$ if fully optically thin. We then derive dust masses of $2.5 \times 10^{-4} M_{\odot}$ at $321 \mathrm{GHz}$ and $1.6 \times 10^{-4} M_{\odot}$ at $658 \mathrm{GHz}$ for the main dust component $\mathrm{C}$, having assumed no photospheric contribution. The lower mass value at $658 \mathrm{GHz}$ is probably due to a combination of optical depth effects and an inaccurate grain emissivity law. We derive dust masses of $4.0 \times 10^{-5} M_{\odot}$ at $321 \mathrm{GHz}$ and $1.8 \times 10^{-5} M_{\odot}$ at $658 \mathrm{GHz}$ for the VY component having subtracting the stellar contribution, which is calculated in Appendix C. The discrepancy in the dust mass values here could be due to an inaccurate grain emissivity law or differences in sensitivities to low surface brightness emission.

\subsection{Dust mass-loss history and mechanisms}

Considering that we find that part of the dust emission around VY CMa arises in compact clumps, we can obtain a new estimate of the average dust mass-loss rate by determining the dust mass that is resolved out in the ALMA observations. We assume the dust to be extended over the $\sim 8^{\prime \prime}$ size of the HST image and use an average dust velocity $V_{\mathrm{d}}$ that depends on grain size and that for VY CMa is $\sim 60 \mathrm{~km} \mathrm{~s}^{-1}$ for grains of $\sim 0.25 \mu \mathrm{m}$ (Decin et al. 2006). We assume an average temperature of $200 \mathrm{~K}$ throughout the shell. The observed VY component arises from the dust lost in the last $\sim 20\left(60 \mathrm{~km} \mathrm{~s}^{-1} / V_{\mathrm{d}}\right) \mathrm{yr}$ and the resolved-out emission $(\sim 1.1 \mathrm{Jy}$ at $321 \mathrm{GHz}$ and $\sim 7.8 \mathrm{Jy}$ at $658 \mathrm{GHz}$ as shown in Fig. B.1) corresponds to the dust lost over $\sim 360\left(60 \mathrm{~km} \mathrm{~s}^{-1} / V_{\mathrm{d}}\right)$ yr before. For $\beta=0.7$ using Eq. (1), we find a dust mass of $\sim 1.8 \times 10^{-3} M_{\odot}$ which is consistent at both frequencies, and a dust mass-loss rate of $5 \times$ $10^{-6}\left(V_{\mathrm{d}} / 60 \mathrm{~km} \mathrm{~s}^{-1}\right) M_{\odot} \mathrm{yr}^{-1}$, which is four times higher than previously estimated by Sopka et al. (1985) and slightly higher than Knapp et al. (1993), taking the different adopted velocities and distances into account. Then, taking all compact emission from the ALMA observations, with the exception of what is around the star, to be in optically thin clumps at $\sim 450 \mathrm{~K}$, those clumps contain $\sim 3 \times 10^{-4} M_{\odot}$ of dust. This is a lower limit, because a significant component of cold dust can potentially be present in C and in the SW and other HST features not detected by ALMA. We can thus conclude that $>17 \%$ of the dust mass around VY CMa is located in clumps ejected within a more quiescent, roughly spherical stellar wind. This picture of a quiescent mass loss disrupted with periods of more localized intense ejections of mass is consistent with the findings of Humphreys et al. (2007) for VY CMa and Ohnaka (2014) for Antares (M1 Iab).
It has been hypothesized that large convection cells initiate mass loss in RSGs by lifting material out to distances beyond the photosphere where dust can form and drive mass loss (e.g., Lim et al. 1998). These convection cells are predicted to have time scales of $\sim 150$ days (Schwarzschild 1975) and are expected to eject material at random PAs from the star, generating supersonic motions and shocks in the process (Lion et al. 2013). Our observations have revealed a massive dust clump centered at $60 R_{\star}$ and a weaker continuous dust feature extending $90 R_{\star}$ to the North. These features imply a continuous directed dust massloss from the star over the past 30-50 yr. This contrasts with what would be expected from mass loss that is only driven by convection, which on these spatial scales would show many dust clumps at random PAs. A mass-loss mechanism that is localized but much more stable over time (i.e., over a few decades) is required to explain our observations. This would suggest a magnetic origin. Indeed a surface magnetic field strength of $10^{3} \mathrm{G}$ has been extrapolated from circumstellar maser measurements for VY CMa (Vlemmings et al. 2002). Cool spots on the photosphere due to localized long-lived MHD disturbances could then enhance local dust formation, and hence drive mass loss in the localized directions that are evident in our ALMA data. Such a mass-loss mechanism would still appear as random mass ejections on larger spatial scales and could be the source of the arcs and clumps seen in VY CMa's outer circumstellar environment by Smith et al. (2001) and Humphreys et al. (2007).

Acknowledgements. This paper makes use of the following ALMA data: ADS/JAO.ALMA\#2011.0.00011.SV. ALMA is a partnership of ESO (representing its member states), NSF (USA) and NINS (Japan), together with NRC (Canada) and NSC and ASIAA (Taiwan), in cooperation with the Republic of Chile. The Joint ALMA Observatory is operated by ESO, AUI/NRAO and NAOJ. EOG and WV acknowledge support from Marie Curie Career Integration Grant 321691 and ERC consolidator grant 614264.

\section{References}

Danchi, W. C., Bester, M., Degiacomi, C. G., Greenhill, L. J., \& Townes, C. H. 1994, AJ, 107, 1469

Decin, L., Hony, S., de Koter, A., et al. 2006, A\&A, 456, 549

Draine, B. T. 2006, ApJ, 636, 1114

Harper, G. M., Brown, A., \& Lim, J. 2001, ApJ, 551, 1073

Herman, J., Burger, J. H., \& Penninx, W. H. 1986, A\&A, 167, 247

Humphreys, R. M. 1974, ApJ, 188, 75

Humphreys, R. M., Helton, L. A., \& Jones, T. J. 2007, AJ, 133, 2716

Jones, T. J., Humphreys, R. M., Helton, L. A., Gui, C., \& Huang, X. 2007, AJ, 133,2730

Kamiński, T., Gottlieb, C. A., Young, K. H., Menten, K. M., \& Patel, N. A. 2013, ApJS, 209, 38

Knapp, G. R., Sandell, G., \& Robson, E. I. 1993, ApJS, 88, 173

Ladjal, D., Justtanont, K., Groenewegen, M. A. T., et al. 2010, A\&A, 513, A53

Lim, J., Carilli, C. L., White, S. M., Beasley, A. J., \& Marson, R. G. 1998, Nature, 392, 575

Lion, S., Van Eck, S., Chiavassa, A., Plez, B., \& Jorissen, A. 2013, in EAS Pub. Ser. 60, eds. P. Kervella, T. Le Bertre, \& G. Perrin, 85

Lipscy, S. J., Jura, M., \& Reid, M. J. 2005, ApJ, 626, 439

Muller, S., Dinh-V-Trung, Lim, J., et al. 2007, ApJ, 656, 1109

Ohnaka, K. 2014, A\&A, 568, A17

Richards, A. M. S., Impellizzeri, C. M. V., Humphreys, E. M., et al. 2014, A\&A, 572, L9

Schwarzschild, M. 1975, ApJ, 195, 137

Shenoy, D. P., Jones, T. J., Humphreys, R. M., et al. 2013, AJ, 146, 90

Shinnaga, H., Moran, J. M., Young, K. H., \& Ho, P. T. P. 2004, ApJ, 616, L47

Smith, N., Humphreys, R. M., Davidson, K., et al. 2001, AJ, 121, 1111

Sopka, R. J., Hildebrand, R., Jaffe, D. T., et al. 1985, ApJ, 294, 242

Vlemmings, W. H. T., Diamond, P. J., \& van Langevelde, H. J. 2002, A\&A, 394, 589

Wittkowski, M., Hauschildt, P. H., Arroyo-Torres, B., \& Marcaide, J. M. 2012, A\&A, 540, L12

Zhang, B., Reid, M. J., Menten, K. M., \& Zheng, X. W. 2012, ApJ, 744, 23

Page 5 is available in the electronic edition of the journal at http://www . aanda.org 
Table A.1. Properties of the two main continuum components, C and VY.

\begin{tabular}{lccccccccccc}
\hline \hline $\mathrm{ID}_{v}$ & $\begin{array}{c}\theta_{\text {maj }} \\
(\mathrm{mas})\end{array}$ & $\begin{array}{c}\theta_{\text {maj }} \\
\left(R_{\star}\right)\end{array}$ & $\begin{array}{c}\theta_{\min } \\
(\mathrm{mas})\end{array}$ & $\begin{array}{c}\theta_{\min } \\
\left(R_{\star}\right)\end{array}$ & $\begin{array}{c}\mathrm{PA} \\
\left({ }^{\circ}\right)\end{array}$ & $\begin{array}{c}S_{v} \\
(\mathrm{mJy})\end{array}$ & $\alpha$ & $\beta$ & $\begin{array}{c}T_{\mathrm{d}} \\
(\mathrm{K})\end{array}$ & $\tau_{v}$ & $\begin{array}{c}M_{\mathrm{d}} \\
\left(M_{\odot}\right)\end{array}$ \\
\hline $\mathrm{C}_{321}$ & $182 \pm 13$ & 33 & $117 \pm 16$ & 21 & $112 \pm 10$ & $348 \pm 16$ & 1.9 & -0.1 & $<450$ & 0.19 & $2.5 \times 10^{-4}$ \\
$\mathrm{C}_{658}$ & $206 \pm 4$ & 37 & $99 \pm 3$ & 18 & $115 \pm 1$ & $1532 \pm 55$ & 1.9 & -0.1 & $<450$ & 0.17 & $1.6 \times 10^{-4}$ \\
$\mathrm{VY}_{321}$ & $219 \pm 19$ & 40 & $144 \pm 33$ & 26 & $4 \pm 17$ & $155 \pm 14$ & 2.7 & 0.7 & 970 & 0.04 & $4.0 \times 10^{-5}$ \\
$\mathrm{VY}_{658}$ & $150 \pm 8$ & 27 & $73 \pm 17$ & 13 & $22 \pm 5$ & $501 \pm 53$ & 2.7 & 0.7 & 970 & 0.02 & $1.8 \times 10^{-5}$ \\
\hline
\end{tabular}

Notes. $\theta_{\text {maj }}$ and $\theta_{\min }$ : major and minor axes of the deconvolved components of the Gaussian fits to the continuum components; PA: position angle; $S_{\nu}$ : integrated flux density from the deconvolved fits; $\alpha$ : spectral index; $\beta$ : dust emissivity spectral index; $T_{\mathrm{d}}$ : dust temperature assuming isothermal and optically thin dust, which may not be the case for the $\mathrm{C}$ component; $\tau_{v}$ : optical depth; $M_{\mathrm{d}}$ : dust mass.

\section{Appendix A: Properties of the two main continuum components}

In Table A.1 we list the properties of the two main continuum components, $\mathrm{C}$ and VY.

\section{Appendix B: Comparison with other (sub-)mm observations}

There have been many previous (sub-)millimeter continuum flux density measurements of VY CMa. In Fig. B.1, we present both the single-dish observations, using the compilations of Knapp et al. (1993) and Ladjal et al. (2010), and the interferometric measurements from this paper and from SMA observations (Shinnaga et al. 2004; Muller et al. 2007; Kamiński et al. 2013). It is immediately apparent that the interferometric observations systematically underestimate the total flux density and can be attributed to flux density that is resolved out by missing short baselines, as well as by having low surface brightness. The MRS at higher frequencies is smaller so more flux density is resolved out, resulting in a larger discrepancy between the single-dish and interferometric measurements. The spectral index determined from the single-dish observations is $\alpha=2.5 \pm 0.2$ and is consistent with our findings in Sect. 3.2 for the optically thin dust. We note that the single-dish data points in Fig. B.1 will also contain flux density from molecular emission lines. Kamiński et al. (2013) find that this accounts for about $25 \%$ of their measured flux density between 279 and $355 \mathrm{GHz}$. This level will vary at the different frequencies and will mainly result in an added upward scatter but should not affect the overall spectral index. Assuming the true spectral index of the interferometric observations to be the same as that of the single-dish observations and assuming there are no significant changes in dust properties on the different scales, we can conclude that the ALMA observations lose $\sim 65 \%$ of the emission at $321 \mathrm{GHz}$ and $\sim 72 \%$ of the emission at $658 \mathrm{GHz}$. Considering the MRS and sensitivity of the ALMA observations, most of the submillimeter dust continuum flux density is thus located in a smooth low surface brightness distribution stretching beyond $\sim 4^{\prime \prime}$, which is consistent with the dust distribution seen over $\sim 8^{\prime \prime}$ with the HST (e.g., Smith et al. 2001).

\section{Appendix C: Stellar flux density contribution}

The stellar flux density contribution $S_{\star}$, needs to be considered when calculating the dust mass from the VY component at these

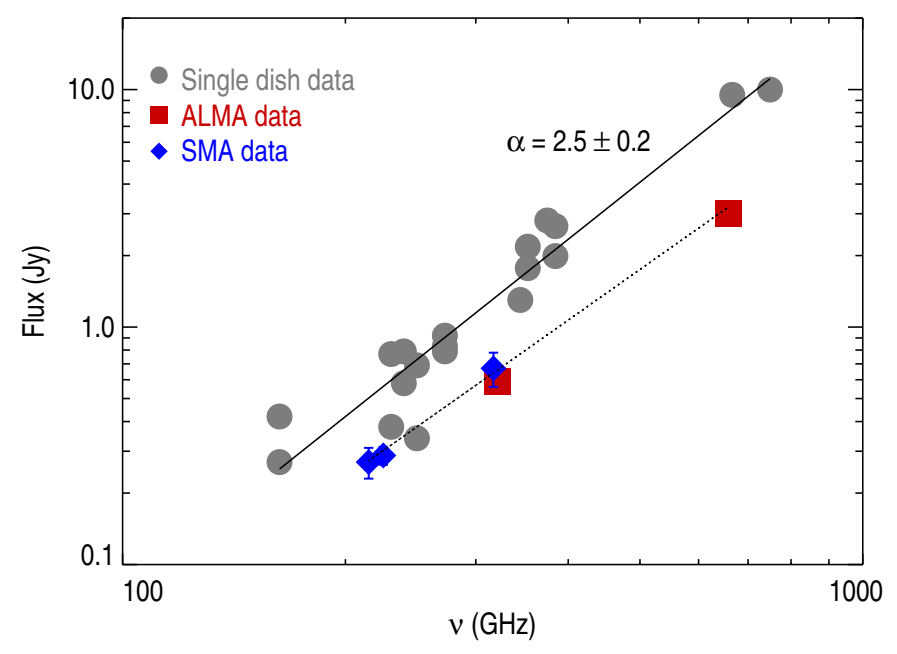

Fig. B.1. Compilation of the single dish bolometer and interferometric $\mathrm{mm} / \mathrm{sub}-\mathrm{mm}$ continuum observations of VY CMa. The gray filled circles are single-dish flux density measurements, while the blue filled diamonds and the red filled squares are interferometric measurements. The solid line indicates the spectral index fit to the single-dish observations, with $\alpha=2.5 \pm 0.2$, while the dotted line is a linear fit to the interferometric observations.

ALMA frequencies. Previous studies have estimated this contribution by calculating the flux density of an optically thick blackbody of radius $R_{\star}$ and temperature $T_{\text {eff. }}$ Estimating the contribution from this method yields $S_{\star}=26.5 \mathrm{mJy}$ at $321 \mathrm{GHz}$ and $S_{\star}=111 \mathrm{mJy}$ at $658 \mathrm{GHz}$, where we have assumed a stellar radius of $R_{\star}=1420 R_{\odot}(6.84 \mathrm{AU}$ or 5.7 mas $)$ and an effective temperature $T_{\text {eff }}=3490 \mathrm{~K}$ (Wittkowski et al. 2012). However, RSGs have weakly ionized extended atmospheres, which will become opaque at (sub-)mm frequencies. To estimate this contribution at these ALMA frequencies, we scaled the Harper et al. (2001) semi-empirical model for the M2 Iab RSG Betelgeuse (without the silicate dust) to the angular diameter of VY CMa. The main source of opacity in this model is the $\mathrm{H}^{-}$and $\mathrm{H}$ free-free opacity from electrons produced by photoionized metals. We then assumed the same ionization fraction and scaled the particle densities so that good agreement was obtained with the Very Large Array centimetre observations of VY CMa from Lipscy et al. (2005). The stellar contribution from this weakly ionized atmosphere is $36 \mathrm{mJy}$ at $321 \mathrm{GHz}$ and $124 \mathrm{mJy}$ at $658 \mathrm{GHz}$. 